\title{
DOI: https://doi.org/10.46296/ig.v4i8edespsep.0029
}

\section{ANÁLISIS DE CRITICIDAD DEL EQUIPAMIENTO INDUSTRIAL DE LA LÍNEA DE BOVINOS DE UN CENTRO DE FAENAMIENTO}

\section{CRITICALITY ANALYSIS OF THE INDUSTRIAL EQUIPMENT OF THE BOVINE LINE OF A SLAUGHTERHOUSE}

\author{
Cedeño-Moreira Wilmer Javier ${ }^{1}$; Gorozabel-Chata Francis Benjamin ${ }^{2}$ \\ ${ }^{1}$ Estudiante de la Maestría en Mantenimiento Industrial, Instituto de Postgrado \\ Universidad Técnica de Manabí, UTM. Portoviejo, Ecuador. Correo: \\ wcedeño3846@utm.edu.ec. ORCID ID: https://orcid.org/0000-0003-4855-5569
}

${ }^{2}$ Máster en Ciencias. Departamento de Ingeniería Mecánica. Universidad Técnica de Manabí, UTM. Portoviejo, Ecuador. ORCID ID: https://orcid.org/0000-0003-1092-3346

\begin{abstract}
Resumen
El mantenimiento es una de las principales estrategias que se aplican en la industria para mantener la continuidad, seguridad, eficacia de los procesos productivos. En este sentido, es imperativo establecer estrategias para mejorar o diseñar los planes de mantenimiento, esto debe hacerse jerarquizando los procesos, sistemas y equipos del proceso productivo. Esto se conoce como análisis de criticidad. El objetivo de este trabajo fue realizar un análisis de criticidad del equipamiento industrial de la línea de bovinos del centro de faenamiento de la ciudad de Portoviejo, Ecuador. Para ello, se conocieron las condiciones del equipamiento industrial y posteriormente se determinó su criticidad. Los resultados mostraron que el centro de faenamiento no posee de un plan de mantenimiento preventivo para el equipamiento industrial, siendo las labores de tipo correctivo, y que los equipos con mayor criticidad son el tecle del izado, tecle para rayado y corte de patas posteriores, sierra eléctrica de cortar canal, sierra de cortar esternón, tecle de descuerado y el noqueador neumático, esto como consecuencia de la frecuencia de fallas presentadas, impacto en la seguridad y costos de mantenimiento. Se sugirió el diseño de un plan de mantenimiento preventivo para los equipos con alta y media criticidad.
\end{abstract}

Palabras clave: bovinos, criticidad, equipos, faenamiento, mantenimiento.

\begin{abstract}
Maintenance is one of the main strategies applied in the industry to maintain the continuity, safety, efficiency of production processes. In this sense, it is imperative to establish strategies to improve or design maintenance plans, this should be done by prioritizing the processes, systems and equipment of the production process. This is known as criticality analysis. The objective of this work was to carry out a criticality analysis of the industrial equipment of the cattle line of the fishing center of the city of Portoviejo, Ecuador. For this purpose, the conditions of the industrial equipment were known and later its criticality was determined. The results showed that the work center does not have a preventive maintenance plan for the industrial equipment, being the corrective type work, and that the equipment most critically is the lifting keypad, keystroke for scratching and cutting of hind legs, electric saw to cut channel, saw to cut sternum, keystroke of descuerado and the pneumatic noqueador, this as consequence of the frequency of presented failures, impact on the safety and maintenance costs. The design of a preventive maintenance plan for equipment with high and medium criticality was suggested.
\end{abstract}

Keywords: cattle, criticality, equipment, slaughter, maintenance.

Información del manuscrito:

Fecha de recepción: 12 de julio de 2021.

Fecha de aceptación: 03 de septiembre de 2021.

Fecha de publicación: 24 de septiembre de 2021. 


\section{Introducción}

El mantenimiento de equipos es una de las principales estrategias que se aplican en la industria con la finalidad de reducir costos. El mantenimiento de los equipos representa un ahorro para la economía de las empresas, en términos de mantener la continuidad, seguridad y eficiencia de los mismos [1], es decir que tiene un impacto en la productividad de cualquier proceso. En este sentido, para cualquier industria, uno de los principales objetivos del área de mantenimiento es identificar y mitigar las fallas del equipamiento industrial, así como atenuar los efectos generados por una máquina dentro de su vida útil o tiempo de operación [1], [2], en otras palabras, lo que se busca es la optimización de los recursos. En este sentido, es imperativo diseñar y establecer estrategias de mantenimiento del equipamiento industrial con la finalidad última de mejorar los indicadores de eficacia y eficiencia de los planes de mantenimiento, esto debe hacerse jerarquizando los equipos de una infraestructura 0 proceso productivo [3]-[5]. Esta jerarquización se conoce como análisis de criticidad.
Un análisis de criticidad permite subdividir los sistemas en secciones que puedan ser manejadas de manera controlada y auditable [6], permitiendo generar una lista ponderada desde el equipo más crítico hasta el menos crítico del total del universo analizado, y además se tiene una clasificación de estos equipos respecto a su criticidad: alta, media y baja [7]. Llegado a este punto el siguiente paso sería el diseño de estrategias para incrementar la productividad de la organización, para lo cual se deberá comenzar con los elementos etiquetados o que son parte de la categoría "alta criticidad", obviamente esto permitirá mejorar el proceso, disminuyendo la frecuencia de la falla 0 el tiempo de permanencia de las mismas, coadyuvando a la mejora del desempeño global de cualquier empresa.

De esta manera, cualquier empresa u organización en la búsqueda para mejorar su proceso productivo puede realizar un análisis de criticidad como requisito primario para mejorar las labores enmarcadas en un plan de mantenimiento. En la literatura se pudo encontrar análisis de criticidad 
en muchas áreas, por ejemplo, para empresas de trasmisión eléctrica [1], para equipos consumidores de energía eléctrica [4], para los sistemas tecnológicos en empresas biofarmacéuticas [6], para análisis de indicadores de gestión en el desarrollo de software de mantenimiento para vehículos livianos [8], para los equipos y sistemas tecnológicos de una termoeléctrica [9], para los equipos de la casa de fuerza de un hospital [10], entre otros. Sin embargo, no se encontró un análisis de criticidad para los equipos de un centro de faenamiento o matadero de animales bovinos.

Por otro lado, el centro de faenamiento (matadero/camal) de la ciudad de Portoviejo, ManabíEcuador, en donde se realiza el sacrificio de animales bovinos y porcinos para obtener su carne en óptimas condiciones para el consumo humano [11]. Este centro de faenamiento fue tecnificado en el año 2010 y remodelado en el año 2017, fecha en la cual se implementaron equipos como: noqueador neumático, polipastos de 1 y 2 toneladas, sierras para cortar esternón, sierra de partir canal y cuartos, entre otros. Sin embargo, debido a que actualmente el mantenimiento que se realiza en el centro de faenamiento es correctivo, este presenta una disminución de su productividad asociadas a las fallas en sus equipos, a los tiempos de reparación de estos, a la deficiencia del número de operarios y al desconocimiento de la funcionalidad de las maquinas.

Por lo descrito anteriormente, en esta investigación se tiene como objetivo realizar un análisis de criticidad del equipamiento industrial de la línea de bovinos del centro de faenamiento de la ciudad de Portoviejo, Manabí-Ecuador para su uso posterior en el diseño de un plan de mantenimiento preventivo.

\section{Materiales y métodos}

La investigación fue de tipo proyectiva, ya que se buscó una solución a la problemática de la gestión de mantenimiento de la línea de bovino del centro de faenamiento de Portoviejo [12]. Para ello, se desarrollaron una serie de actividades que permitieron la recolección de información en el área de interés, pasando desde la observación directa, la entrevista, la 
encuesta, la realización de un análisis de criticidad, y finalmente, se definieron los criterios que deben considerarse en el plan de mantenimiento.

La investigación estuvo enmarcada dentro de un diseño de campo no experimental, caracterizada principalmente porque los datos se recolectaron del área de mantenimiento, del área del departamento de producción, datos que no fueron manipulados por los investigadores [12].

2.1. Proceso de faenamiento de animales bovinos y condiciones actuales del equipamiento industrial del centro de faenamiento.

A través de las técnicas de recolección de información se pudo conocer el proceso de faenamiento de animales bovinos y las condiciones actuales del equipamiento industrial de la línea de bovino del centro de faenamiento de Portoviejo. Se realizaron observaciones directas por parte de los investigadores, encuestas al personal del área de mantenimiento y entrevistas al personal del departamento de producción. Esto permitió -entre otras actividades- conocer que equipo se usa para cada paso del sacrificio de los animales bovinos y constatar que no existe un plan de mantenimiento preventivo en la organización, pudiendo observar la ocurrencia de fallos en los equipos, las paradas no programadas y el tiempo de estas, lo cual hizo posible hacer un registro durante los meses de enero a marzo de 2020.

\subsection{Cálculo de la criticidad del} equipamiento industrial de la línea de bovino del centro de faenamiento.

La metodología para determinar la criticidad se basa en la operación de los equipos y de los registros históricos de las fallas que se presentan, por lo cual se hicieron registros del trimestre enero-marzo de 2020. Este registro debe hacerse para cada equipo y comprende parámetros como las fallas registradas, componentes asociados a la falla, la frecuencia de la falla, el tiempo promedio y total de la falla.

Para determinar la criticidad del equipamiento industrial que presentó fallas en la línea de faenado de bovinos, se aplicaron los criterios establecidos en las normas SAE JA1011 e SAE JA1012, las cuales 
indican que es necesario considerar los factores de frecuencia (FF) y consecuencias (CO) asociadas (impacto operacional, factor flexibilidad operacional, costos de mantenimiento, impacto medio ambiente, impacto seguridad). Finalmente, la criticidad representa el producto entre el factor de frecuencia y la sumatoria de las consecuencias. Una metodología similar a la empleada en esta investigación fue utilizada por [13],
[14]. Los valores para los factores FF y CO se muestran en la Tabla 1.

Una vez obtenido el producto de la frecuencia de falla (FF) y las consecuencias (CO) se obtiene el valor de criticidad (CT), este valor se calcula para cada equipo y debe ubicarse en la matriz de criticidad dentro de un rango de colores y valores que se muestran en la figura 1.

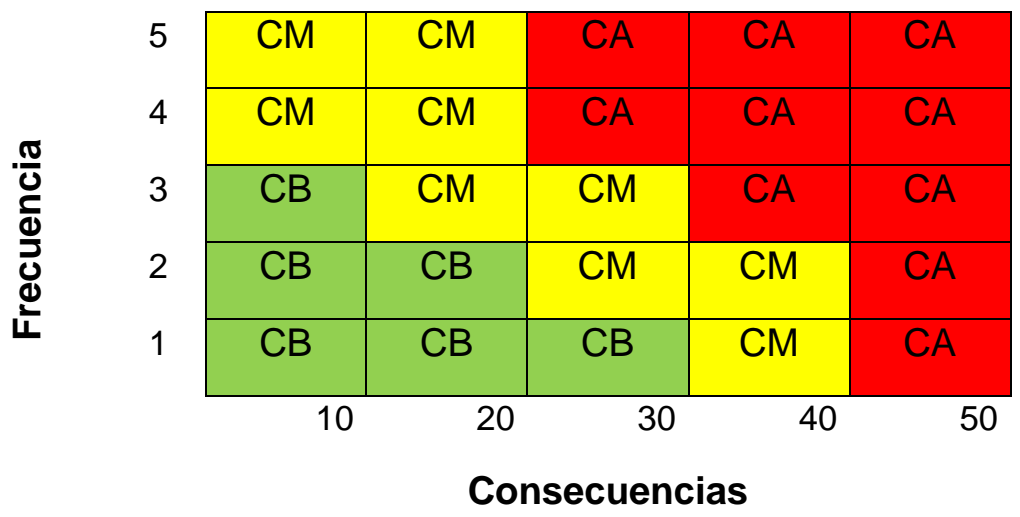

Criticidad alta, color rojo, valores $50 \leq \mathrm{CT} \leq 125$; Criticidad media, color amarillo, valores $30 \leq \mathrm{CT} \leq 49$; Criticidad baja, color verde $5 \leq \mathrm{CT} \leq 29$

Fig. 1. Matriz de criticidad

Tabla 1. Factores de frecuencia y consecuencia [14]

\begin{tabular}{cc}
\hline Factor de frecuencia (FF) & \\
\hline Descripción & $\mathbf{P}^{\star}$ \\
Frecuente, Mas de 3 eventos al año & 5 \\
Probable, 1-3 eventos al año & 4 \\
Posible, 1 evento en 3 años & 3 \\
Improbable, 1 evento en 5 años & 2 \\
Muy improbable, menos de un evento en 5 años & 1 \\
\hline Factores de consecuencias (CO) & \\
\hline Impacto operacional (IO) & $\mathbf{P}^{\star}$ \\
\hline Perdidas mayores 75\% producción mes & 5 \\
Perdidas 50\% a 74\% producción mes & 4 \\
Perdidas 25\% a 49\% producción mes & 3 \\
Perdidas 10\% a 24\% producción mes & 2
\end{tabular}


Perdidas inferiores $10 \%$ producción mes

Factor flexibilidad operacional (FO)

No existe stock, tiempos reparación altos

Stock parcial, procedimiento reparación complejo

Stock parcial, procedimiento reparación sencillo

Stock Suficiente, procedimiento reparación complejo

Stock suficiente, tiempos reparación bajos

\begin{tabular}{cc}
\hline Costos de mantenimiento (CM) & $\mathbf{P}^{*}$ \\
\hline Costos materiales superior 20000 USD & 5 \\
Costos materiales superior 10000-20000 USD & 4 \\
Costos materiales superior 3000-10000 USD & 3 \\
Costos materiales superior 200-3000 USD & 2 \\
Costos materiales inferior 200 USD & 1 \\
\hline Impacto medio ambiente (IMA) & $\mathbf{P}^{*}$ \\
\hline Daños irreversibles en el ambiente & 5 \\
Daños severos al ambiente & 4 \\
Daños medios al ambiente & 3 \\
Daños mínimos al ambiente & 2 \\
Sin daño ambiental & 1 \\
\hline Impacto seguridad (IS) & $\mathbf{P}^{*}$ \\
\hline Muerte o incapacidad & 5 \\
Incapacidad parcial o permanente & 4 \\
Daños o enfermedades severas & 3 \\
Daños leves en personas & 2 \\
Sin impacto en la seguridad & 1 \\
\hline
\end{tabular}

1

\section{5}

4

3 
estancia regulado por la ley, debiendo ser hidratadas, además de descansar y relajación muscular [11], seguidamente se les realiza control veterinario.

Posteriormente, los bovinos que se diagnostiquen sanos pasan a la sección de noqueo e insensibilización, lo cual se realiza a través de un noqueador (pistola) neumático, insensibilizando a la res a ser sacrificada y evitándole un sufrimiento mayor en el degüello. Luego al animal se le cortan las extremidades y es izado o elevado empleando polipasto de 2 Ton. En esta parte del proceso, la res es colgada de los cuartos traseros, en un gancho que está unido a un riel, lo cual facilita su movilidad en el proceso de desangrado y los pasos siguientes del faenado [11].

Posterior al izado, se realiza el degüello, donde al animal que se encuentra boca abajo se le realiza un corte en las arterías del cuello, lo cual facilita el desangramiento. Seguidamente, se realiza el corte de la cola y extremidades posteriores (corte de patas y cabeza).
Inmediatamente, al animal se le realiza el rayado de cuero, siendo esto un corte superficial en la piel, realizado normalmente con un cuchillo. Luego se procede con el descuerado o desollado del animal, donde se retira la piel del animal empleando un polipasto (tecle) de 1 tonelada aplicando fuerza.

Luego se procede con el corte del esternón o fisurado, donde se realiza una incisión longitudinal del esternón y la columna vertebral, que se realiza sobre el animal faenado, utilizando una sierra eléctrica.

Se avanza con el proceso de eviscerado, donde se extraen los órganos del animal faenado. Esto se realiza con un polipasto de 1 tonelada. Finalmente, el proceso termina con el despiece y el despacho de la carne para su posterior comercialización. La figura 2 muestra el diagrama de proceso del faenamiento de bovinos. 

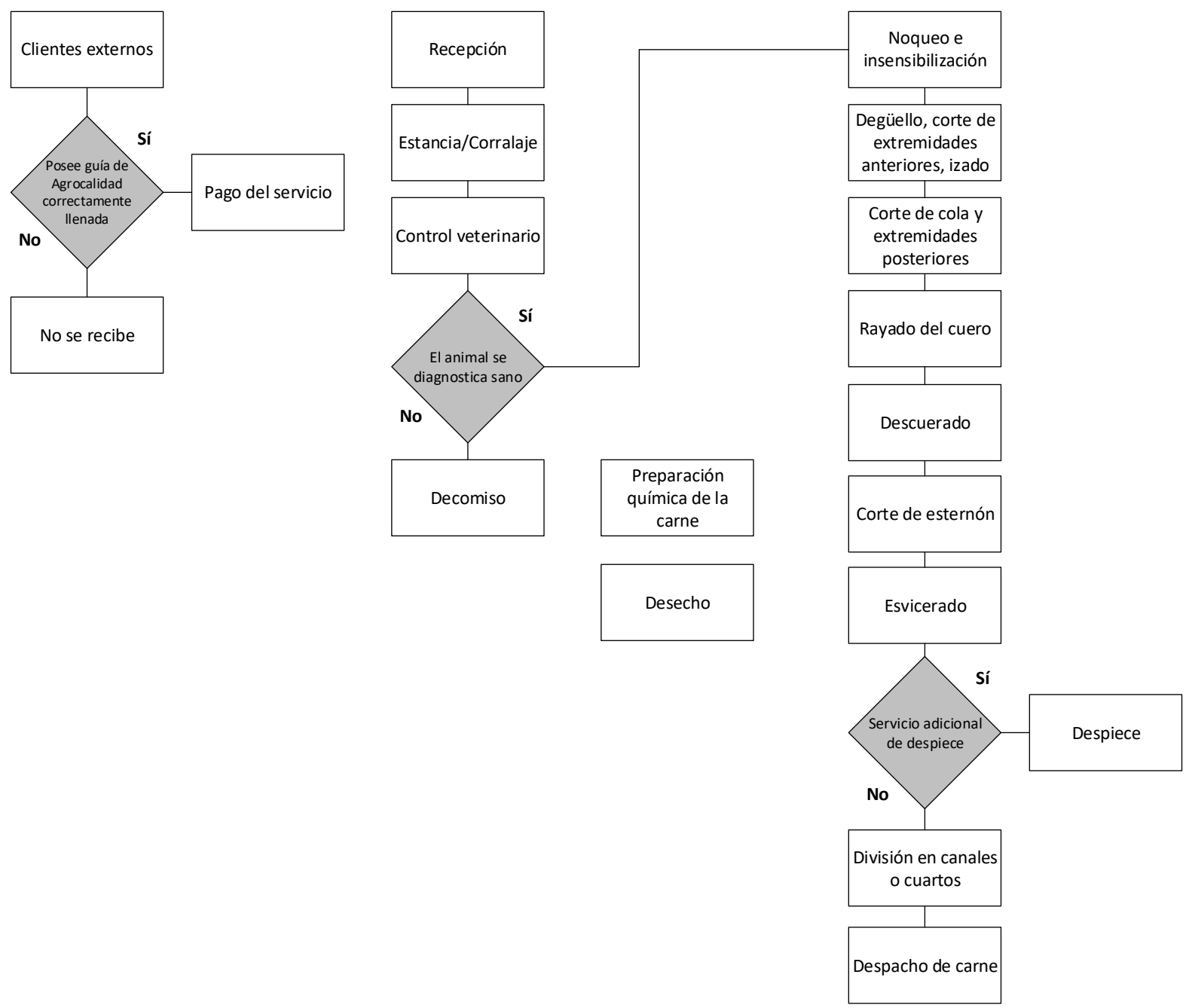

Fig. 2. Diagrama de proceso para el faenado de bovinos.

Fuente: Centro de faenamiento de Portoviejo.

Conocido el proceso de total se recolectó información de un faenamiento, se procedió a evaluar total de 5 personas que laboran en el las condiciones de mantenimiento centro de faenamiento de Portoviejo. del equipamiento de la línea de bovino. Para ello, se recolecto información a través de la observación directa del proceso de faenado, la encuesta aplicada a todo el personal del área de mantenimiento (3 trabajadores) y entrevista al personal del departamento de producción (2 trabajadores: jefe y supervisor). En

Mediante la observación directa se constató a priori que no existe un plan de manteniendo preventivo, por lo que las labores de mantenimiento que se realizan son correctivas, es decir, se realizan una vez que la falla se ha presentado. Por otra parte, la percepción del personal del área de mantenimiento se le aplicó una 
encuesta compuesta por preguntas cuyas respuestas eran dicotómicas. Las preguntas estaban vinculadas a la línea de bovino del centro de faenamiento. A través de la encuesta, el personal de mantenimiento expresa de manera unánime lo siguiente:

- Las labores de mantenimiento están contempladas dentro de un programa, que son planificadas y clasificadas de acuerdo con su finalidad (inspecciones rutinarias, lubricación, sustitución de piezas, recambios, entre otros), que se estima el tiempo requerido para realizar las labores de mantenimiento, y además considera la ejecución de actividades de mantenimiento preventivo es una alternativa para el equipamiento industrial.

- No se mide el tiempo de paradas no programadas debido a labores de mantenimiento realizadas, tampoco existe un registro de las herramientas, repuestos y suministros requeridos para el mantenimiento del equipamiento industrial.
Por otro lado, la mayoría del personal de mantenimiento (66 \%) expresa lo siguiente:

- Se llevan a cabo evaluaciones preliminares para determinar las condiciones de funcionamiento del equipamiento industrial y que son analizadas las fallas y sus efectos en la búsqueda de actividades de mantenimiento.

- El personal no cuenta con especificaciones del fabricante para abordar las fallas del equipamiento industrial.

Ahora, como se indicó previamente, el personal del departamento de producción fue entrevistado, y a partir de esto se pudo conocer sus impresiones respecto al mantenimiento que se realiza en la línea de bovinos, siendo estas las siguientes:

- No se lleva un registro de las paradas no programadas por fallas en el equipamiento.

- Las paradas por fallas no programadas han afectado la producción, pero tampoco llevan un registro. 
- Cuando ocurre una falla, el personal de mantenimiento la soluciona.

- Falta personal en el área de mantenimiento.

- Es necesario un plan de mantenimiento preventivo.

Es necesario destacar que, con base a las fallas observadas y registradas en el centro de faenamiento, así como la información recolectada del personal que allí labora, se evidenció que las actividades de mantenimiento son de naturaleza correctiva, por ende, se ejecutaron después que la falla o avería ocurrió.

Por otra parte, el personal señaló que no cuenta con especificaciones del fabricante para abordar las fallas del equipamiento industrial y consecuentemente, las labores de mantenimiento se realizan de una manera empírica y luego que la falla ocurre. Otra situación a considerar es que no se lleva un registro de las fallas que ocurren en el centro de faenamiento, así como el tiempo que permanecen ni el impacto que causa en la producción. En este sentido, es necesario que desde la gestión de mantenimiento se pueda conseguir el nivel máximo de efectividad en el funcionamiento del proceso productivo con la menor contaminación del ambiente y mayor seguridad al menor costo posible [4].

\subsection{Cálculo de la criticidad.}

Aunque normalmente no se lleva un registro de las fallas presentadas por el equipamiento, para la realización de esta investigación se realizó tal acción durante el trimestre eneromarzo 2020 son los que se muestran en la Tabla 2.

Tabla 2. Fallas registradas en el trimestre enero-marzo 2020.

\begin{tabular}{|c|c|c|c|c|c|c|c|c|c|c|}
\hline \multirow{3}{*}{$\begin{array}{l}\text { Fallas } \\
\text { registradas }\end{array}$} & \multirow[b]{3}{*}{$\begin{array}{l}\text { Componentes } \\
\text { asociados a } \\
\text { la falla }\end{array}$} & \multicolumn{3}{|c|}{ Enero } & \multicolumn{3}{|c|}{ Febrero } & \multicolumn{3}{|c|}{ Marzo } \\
\hline & & \multicolumn{3}{|c|}{ Tiempo. } & \multicolumn{3}{|c|}{ Tiempo. } & \multicolumn{3}{|c|}{ Tiempo. } \\
\hline & & Frec & Prom. & Tot. & Frec & Prom. & Tot. & Frec. & Prom. & Tot. \\
\hline \multirow{4}{*}{$\begin{array}{l}\text { Falla de la } \\
\text { pistola del } \\
\text { noqueador } \\
\text { neumático }\end{array}$} & Amortiguador & 1 & 0:30:00 & $0: 30: 00$ & -- & -- & -- & -- & -- & -- \\
\hline & Perno percutor & 1 & $0: 45: 00$ & 0:45:00 & -- & -- & -- & 1 & $0: 40: 00$ & $0: 40: 00$ \\
\hline & $\begin{array}{l}\text { Tuerca de } \\
\text { sujeción }\end{array}$ & 2 & 0:30:00 & $1: 00: 00$ & 5 & $0: 30: 00$ & $2: 30: 00$ & 2 & $0: 40: 00$ & $1: 20: 00$ \\
\hline & O-ring & 2 & 0:25:00 & $0: 50: 00$ & -- & -- & -- & 2 & 0:23:00 & 0:46:00 \\
\hline
\end{tabular}




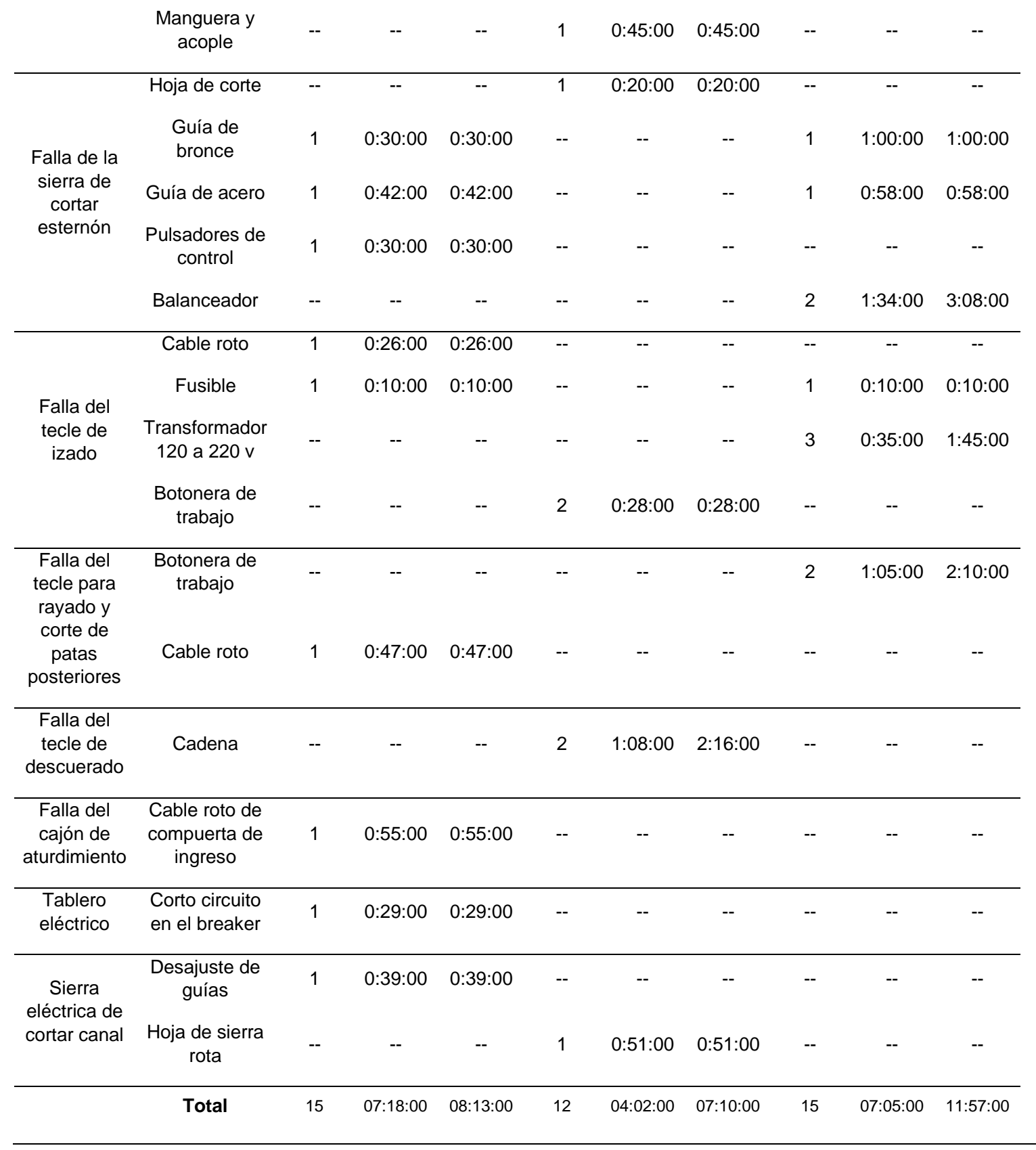

Los equipos que presentaron fallas fueron los siguientes: el noqueador neumático, la sierra de cortar esternón, la sierra eléctrica para cortar canal, el tecle de izado, el tecle para rayado y corte de patas posteriores, el tecle de descuerado, el cajón de aturdimiento y el tablero eléctrico.
Una relación gráfica de la frecuencia de falla, el tiempo promedio y total (horas) de la falla y el mes de ocurrencia se muestra en la figura 3. Comparando el mes de enero con el de marzo se observa que, aunque la frecuencia de falla permanece constante (15), el número de horas totales de permanencia de la falla 
pasa de 8:13:00 a 11:57:00, lo cual se debe a que se necesitó un mayor tiempo de respuesta del personal de mantenimiento para solucionar la falla, debido a que el mes de enero se presentaron solamente desajustes de las piezas, mientras que ya en el mes de marzo, las piezas experimentaron daños, por lo que se necesitaron repuestos que no se tienen en stock, traduciéndose en tiempos más largos de reposición y reparación, así como también en costos mayores.

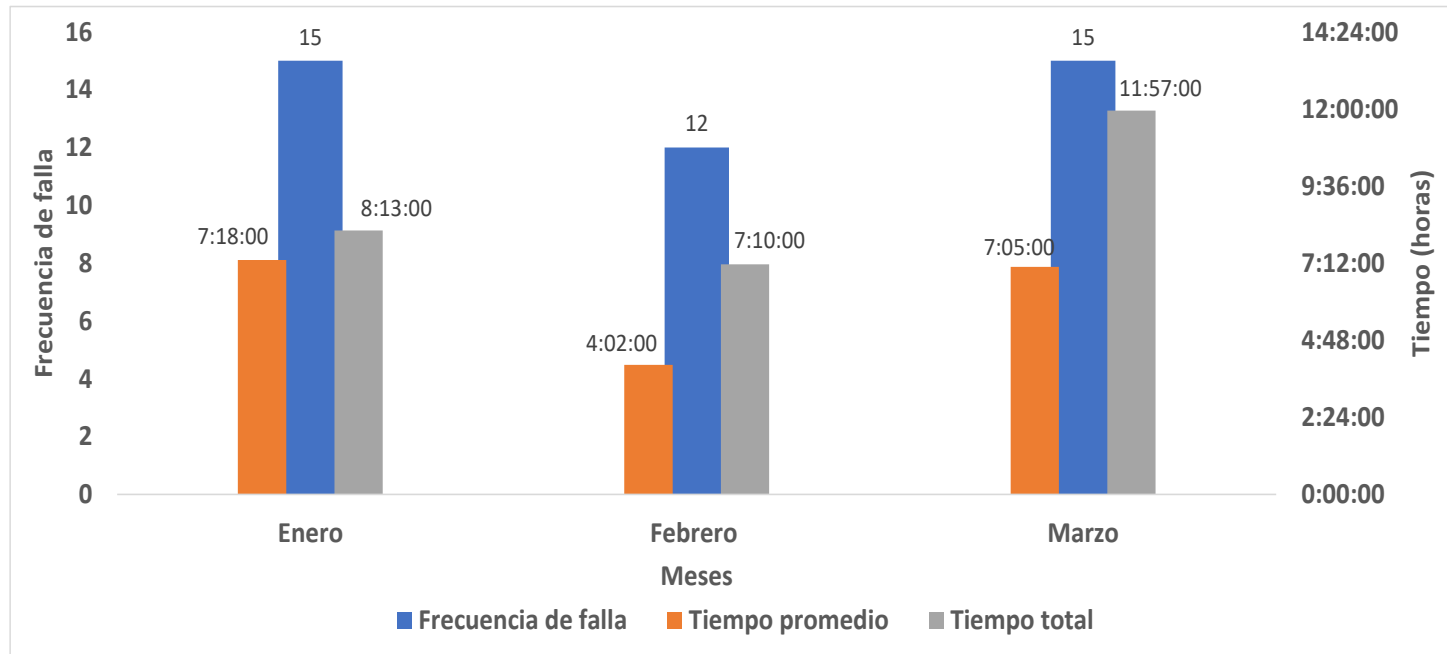

Fig. 3. Frecuencia de falla versus tiempo de promedio y total de la totalidad de las fallas registradas en la línea de faenamiento de bovinos durante el trimestre enero-marzo 2020.

Para determinar la criticidad (CT) del equipamiento industrial que presentó fallas en la línea de faenado de bovinos, se aplicaron las normas SAE JA1011 y SAE JA1012, las cuales indican que es necesario considerar los factores de frecuencia (FF) y consecuencias (CO) asociadas (impacto operacional, factor flexibilidad operacional, costos de mantenimiento, impacto medio ambiente, impacto seguridad), las cuales fueron mostradas previamente en la tabla 1. Sin embargo, en el centro de faenamiento de Portoviejo, no existe un registro de los impactos operacionales que producen las fallas, por lo cual no se pudo aplicar la norma de manera expedita, y se plantearon los impactos operacionales en función de una estimación del porcentaje de pérdidas de producción mensual, esto se realizó de manera similar a [14]. La criticidad (CT) se calculó a partir de la ponderación los factores de frecuencia y consecuencia estipulados en la tabla 1 , el procedimiento consistió en asignar la 
ponderación al factor de frecuencia (FF) y consecuencias (CO), esta última está subdividida en: impacto operacional (IO), factor flexibilidad operacional (FO), costos de mantenimiento $(\mathrm{CM})$, impacto medio ambiente (IMA), impacto seguridad (IS). Finalmente, la criticidad (CT) representa el producto entre el factor de frecuencia y la sumatoria de las consecuencias (Tabla 3). En la tabla mencionada, el total de fallas (TF) corresponde a la sumatoria de fallas registrados en el trimestre eneromarzo de 2020, número que puede ser verificado sumando los fallos mostrados en la tabla 2.
La ponderación de cada factor se realizó con base en los factores de frecuencia y consecuencias mostrados en la tabla 1 y tomando en cuenta de criterios de expertos en el área de mantenimiento, dando como resultado los valores de CT. De esta manera, los equipos con una alta criticidad son los que tienen valores de CT entre 50-125 y se resaltan en rojo, los equipos con criticidad media son los que tienen valores entre 30-49 y se encuentran resaltados en amarillos, y finalmente, los equipos con CT entre 5-29 y resaltados en verdes tienen criticidad baja.

Tabla 3. Criticidad jerarquizada de los equipos del centro de faenamiento.

\begin{tabular}{cccccccccc}
\hline Equipo & TF & FF & IO & FO & CM & IMA & IS & CO & CT \\
\hline Tecle del izado & 7 & 5 & 5 & 5 & 2 & 1 & 4 & 17 & 85 \\
$\begin{array}{c}\text { Polipasto para rayado y } \\
\text { corte de patas posteriores }\end{array}$ & 3 & 5 & 5 & 5 & 2 & 1 & 4 & 17 & 85 \\
Sierra eléctrica de cortar & 2 & 5 & 4 & 5 & 2 & 1 & 3 & 15 & 75 \\
$\quad$ canal & 8 & 5 & 3 & 5 & 2 & 1 & 3 & 14 & 70 \\
Sierra de cortar esternón & 2 & 5 & 2 & 5 & 2 & 1 & 4 & 14 & 70 \\
Tecle de descuerado & 17 & 5 & 1 & 5 & 2 & 1 & 3 & 12 & 60 \\
Noqueador neumático & 1 & 4 & 3 & 5 & 2 & 1 & 1 & 12 & 48 \\
Tablero eléctrico & 1 & 4 & 3 & 5 & 1 & 1 & & 10 & 40 \\
Cajón de aturdimiento & 0 & 1 & 4 & 5 & 2 & 1 & 3 & 15 & 15 \\
Sierra de cortar cuartos & 0 & 1 & 4 & 5 & 2 & 1 & 2 & 14 & 14 \\
Tecle del izado de vísceras & 0 & 1 & 2 & 4 & 2 & 1 & 1 & 10 & 10 \\
Compresor & 0 & 1 & 2 & 2 & 1 & 1 & 1 & 7 & 7
\end{tabular}




\begin{tabular}{|c|c|c|c|c|c|c|c|c|c|}
\hline Bomba 2 hp & 0 & 1 & 2 & 2 & 1 & 1 & 1 & 7 & 7 \\
\hline Bomba 3 hp & 0 & 1 & 2 & 2 & 1 & 1 & 1 & 7 & 7 \\
\hline Bomba $4 \mathrm{hp}$ & 0 & 1 & 2 & 2 & 1 & 1 & 1 & 7 & 7 \\
\hline
\end{tabular}

Clasificación: Criticidad alta, color rojo, valores $50 \leq \mathrm{CT} \leq 125$; Criticidad media, color amarillo, valores $30 \leq \mathrm{CT} \leq 49$; Criticidad baja, color verde $5 \leq \mathrm{CT} \leq 29$

Los equipos a los que se les debe prestar atención para mejorar los índices de eficiencia y eficacia del proceso productivo de la línea de bovino del centro de faenamiento de Portoviejo son: el tecle del izado, tecle para rayado y corte de patas posteriores, sierra eléctrica de cortar canal, sierra de cortar esternón, tecle de descuerado y el noqueador neumático, pudiendo observar que estos equipos son imprescindibles para el desarrollo pleno del proceso productivo del faenado de bovinos sin que haya perdidas en la producción por paradas no programas ocasionadas por fallos de estos equipos.

A partir del análisis de criticidad se plantea al centro de faenamiento de Portoviejo la implementación de un programa de mantenimiento preventivo para los equipos de alta y media criticidad, lo cual constituye una investigación en curso, sin embargo, en primera instancia, se pueden sugerir algunas medidas 0 consideraciones que deben ser incluidas en dicho plan. De esta manera, se propone: llevar un registro detallado de los fallos que se presenten en el equipamiento, en conjunto con el departamento de producción se debe determinar o por lo menos estimar las perdidas en la producción de acuerdo al tiempo de las paradas no programadas, se debe crear un stock de repuestos con mayor frecuencia de reposición. También se proponen medidas como aumento del personal que labora en el área de mantenimiento y su capacitación constante. Se sugiere comprar equipos que vengan con manuales de uso y mantenimiento y de ser necesario, para los equipos que demanden un mantenimiento muy específico o complicado, se recomienda subcontratar el servicio. Para los equipos con baja criticidad se sugiere realizar planes de mantenimiento correctivos. 


\section{Conclusiones}

A través de las técnicas de recolección de información se pudo constatar que el centro de faenamiento de la ciudad de Portoviejo en Manabí, Ecuador realizan labores de mantenimiento correctivas, que no se lleva un registro de las paradas no programadas a causa de fallos en los equipos ni tampoco un registro del impacto en la producción.

El análisis de criticidad realizado a los equipos existentes permitió determinar los equipos con alta, media y baja criticidad. Los equipos con una alta criticidad son el tecle del izado, tecle para rayado y corte de patas posteriores, sierra eléctrica de cortar canal, sierra de cortar esternón, tecle de descuerado y el noqueador neumático. Los de criticidad media son el cajón de aturdimiento y tablero eléctrico, y finalmente, los de baja criticidad son la sierra de cortar cuartos, las bombas y el compresor. Con base en el análisis de criticidad se sugirió un plan de mantenimiento preventivo para los equipos con alta y media criticidad.

\section{Bibliografía}

[1] A. Díaz-Concepción, L. VillarLedo, J. Cabrera-Gómez, A. Gil-Henríquez, R. MataAlonso, and RodríguezPiñero, "Implementación del Mantenimiento Centrado en la confiabilidad en empresas de trasmisión eléctrica," Ing. Mecánica, vol. 19, no. 3, pp. 137-142, 2016.

[2] A. Díaz Concepción, A. Del Castillo Serpa, and L. Villar Ledo, "Instrumento para evaluar el estado de la gestión de mantenimiento en plantas de bioproductos: Un caso de estudio," Ingeniare. Rev. Chil. Ing., vol. 25, no. 2, pp. 306313, 2017.

[3] I. Estándar, "Norma SAE JA1011. Criterios de Evaluación para Procesos de Mantenimiento Centrado en Confiabilidad. Norma para vehículos aeroespaciales y de superficie.," 1999.

[4] J. Espinosa-Martínez, E. PazMartínez, R. PérezBermudez, and I. AcostaPérez, "Contribución del mantenimiento centrado en la confiabilidad para el estudio de fallos a equipos consumidores de energía eléctrica," Rev. Cent. Azúcar, vol. 47, no. 1, pp. 22-32, 2020.

[5] M. Viscaíno-Cuzco, S. Villacrés-Parra, C. GallegosLondoño, and $\mathrm{H}$. Negrete- 
Costales, "Evaluación de la gestión del mantenimiento en hospitales del Instituto Ecuatoriano de Seguridad Social de la Zona 3 del Ecuador," INGENIUS, pp. 5971, 2019, doi: https://doi.org/10.17163/ings. n22.2019.06.

[6] A. Enriques-Gaspar, A. DíazConcepción, L. Villar-Ledo, A. Del Castillo-Serpa, A. Rodríguez-Piñero, and $A$. Alfonso-Álvarez, "Tecnología para el análisis de criticidad de los sistemas tecnológicos en empresas biofarmacéuticas," Ing. Mecánica, vol. 23, no. 1, pp. 1-11, 2020.

[7] A. Del Castillo-Serpa, M. Brito-Ballina, and E. FragaGuerra, "Análisis de criticidad personalizados," Ing. Mecánica, vol. 12, no. 3, pp. 1-12, 2009.

[8] B. A. Cuaical-Angulo, L. F. Buenaño-Moyano, and $\mathrm{L}$. Moreno-Pinduisaca, "Análisis de indicadores de gestión CMD en el desarrollo de software de mantenimiento para vehículos livianos," FIPCAEC, vol. 4, no. 2, pp. 145-170, 2019, doi: AEC (núm. 2) Vol. 4, Año 4 Diciembre Edición Especial 2019, pp. 145-170 DOI: https://doi.org/10.23857/fipca ec.v4i4.128 Análisis.

[9] A. Díaz-Concepción, A. del Castillo-Serpa, M. Toledo-
García, and J. CabreraGómez, "Obtención de un modelo de criticidad para los equiposy sistemas tecnológicos de unatermoeléctrica," Ing. Energética, vol. 37, no. 3, pp. 217-227, 2016, [Online]. Available:

https://www.redalyc.org/articu lo.oa?id=329147536006.

[10] S. J. Cossios Risco and J. L. Arévalo Daza, "Gestión del mantenimiento para incrementar la confiabilidad en los equipos de la casa de fuerza de un hospital," INGnosis, vol. 4, no. 2, pp. 159-169, 2018.

[11] EMRAQ-EP, "Proceso de faenamiento de Bovinos," Empresa Pública Metropolitana Rastro de Quito, 2020. http://www.epmrq.gob.ec/inde x.php/servicios/faenamiento/f aenamiento-bovinos.

[12] J. Hurtado De Barrera, Metodología de la Investigación: guía para una comprensión holística de la ciencia, 4ta Ed. Caracas: Quirón Ediciones, 2010.

[13] L. Vargas Henríquez, S. Mejía Ruiz, and C. Vásquez Caballis, "Metodología para diagnosticar fallas localizadas en equipos de una línea de producción de carbón activado," INGENIARE, vol. 24, pp. 77-88, 2018, doi: 
https://doi.org/10.18041/1909

-2458/ingeniare.24.5173.

[14] J. Ramirez and H. Moreno, "Elaboración de un análisis de criticidad y disponibilidad para la atracción $x$-treme del parque Mundo Aventura, tomando como referencia las normas, SAE JA1011 y SAE JA1012," Universidad Distrital Francisco José de Caldas, 2017. 